\title{
The quantum Zeno paradox, 42 years on*
}

\author{
Wayne M. Itano** \\ Time and Frequency Division, National Institute of Standards and Technology, Boulder, CO 80305, USA
}

The term 'quantum Zeno paradox' or 'quantum Zeno effect' refers to the slowing down of the evolution of a quantum system as it is observed more and more frequently. In 1977, Misra and Sudarshan gave a theoretical demonstration of its existence. There has been disagreement regarding the definition of the effect and whether it is really a paradox, requiring new physics, or a consequence of ordinary quantum mechanics. The experiment of Itano, Heinzen, Bollinger and Wineland, published in 1990, is the one most often called a demonstration of the quantum Zeno effect. Some different interpretations are discussed.

\section{Introduction}

ACCORDING to Wikipedia, an Internet-based encyclopaedia, the quantum Zeno effect is defined as follows:

'The quantum Zeno effect (also known as the Turing paradox) is a feature of quantum-mechanical systems allowing a particle's time evolution to be arrested by measuring it frequently enough with respect to some chosen measurement setting, ${ }^{1}$.

This definition avoids the use of the problematic term 'wavefunction collapse', sometimes used in this context. That term is unnecessary and can lead to confusion. 'Measurements', in this context, should be understood as interactions with an external system that disturb the unitary evolution of the quantum system in a way that is effectively like a projection operator. A slowing of the time evolution, as opposed to a complete freezing, is generally regarded as a demonstration of the quantum Zeno effect.

\section{The 1977 Misra and Sudarshan paper}

Misra and Sudarshan ${ }^{2}$, in an article published in 1977, studied the evolution of a quantum system subjected to frequent ideal measurements. They showed that, in the limit of infinitely frequent measurements, a quantum system remains in its initial state. Applied to the case of an unstable particle whose trajectory is observed in a bubble chamber or film emulsion, this result seemed to imply

*Work of the US Government. Not subject to US copyright.

**e-mail: wayne.itano@nist.gov that such a particle would not decay, in contradiction to experiment. In this particular case, the resolution to the apparent paradox lies in the fact that the interactions between the particle and its environment that lead to the observed track are not sufficiently frequent to modify the lifetime of the particle.

Figure 1 shows the time distribution of the literature citations to the article by Misra and Sudarshan ${ }^{2}$. The total number of citations listed in the Web of Science database $^{3}$ in early November 2018 was 1351. The graph shows a relatively low but slowly increasing number of citations per year for about a decade, followed by larger increases, continuing, with some fluctuations, up to the present. A similar graph, published in an earlier version of this article ${ }^{4}$, ended in 2006. It seemed to show a decrease in the number of citations per year. In fact, an extrapolation of the trend of the previous several years would have predicted the frequency of citations dropping to zero by about 2019. However, that trend has reversed, although it shows signs of levelling off at a higher level. Part of the recent increase in the number of citations per year could be due to increased interest in quantum information processing, where the quantum Zeno effect might find practical applications.

\section{Simple derivation of the quantum Zeno effect}

The quantum Zeno effect can be derived in an elementary way by considering the short-time behaviour of the state vector $^{5}$. (The treatment of Misra and Sudarshan ${ }^{2}$ is more general, since it involves the density matrix.) Let $|\phi\rangle$ be the state vector at time $t=0$. If $H$ is the Hamiltonian, in units where $\hbar=1$, then the state vector at time $t$ is $\exp (-i H t)|\phi\rangle$, and the survival probability is $S(t)=$ $\mid\left\langle\phi\left|\exp \left(-\left.i H t|\phi\rangle\right|^{2}\right.\right.\right.$. If $t$ is small enough, it should be possible to make a power series expansion:

$$
\exp (-i H t) \approx I-i H t-\frac{1}{2} H^{2} t^{2} \ldots
$$

so that the survival probability is

$$
S(t)=|\langle\phi|\exp (-i H t)| \phi\rangle|^{2} \approx\left[1-(\Delta H)^{2} t^{2}\right]
$$

where

$$
(\Delta H)^{2} \equiv\left\langle\phi\left|H^{2}\right| \phi\right\rangle-\langle\phi|H| \phi\rangle^{2} .
$$




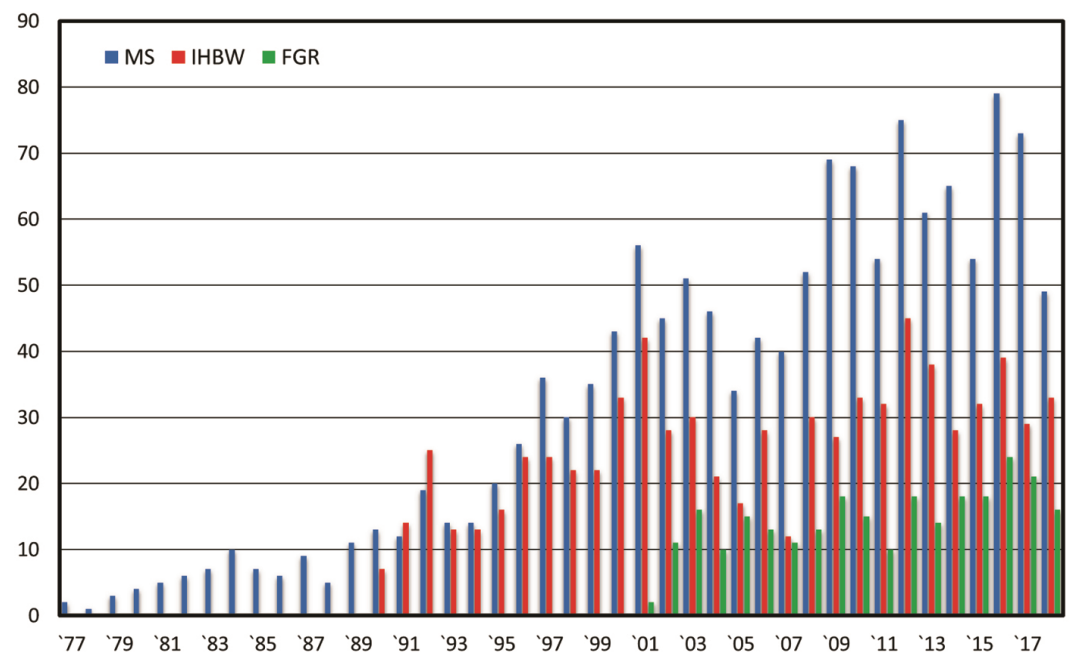

Figure 1. Graph of the number of citations per year to Misra and Sudarshan ${ }^{2}$ (MS) in blue, Itano et al. ${ }^{6}$ (IHBW) in red and Fischer et al. ${ }^{8}$ (FGR) in green, from 1977 through late 2018.

Many quantum systems have states whose survival probability appears on ordinary timescales to be a decreasing exponential in time. This is inconsistent with the quadratic time dependence of eq. (1) and implies that in such cases eq. (1) holds only for very small $t$. Consider the survival probability $S(T)$, where the interval $[0, T]$ is interrupted by $n$ measurements at times $T / n, 2 T / n, \ldots, T$. Ideally, these measurements are instantaneous projections and the initial state $|\phi\rangle$ is an eigenstate of the measurement operator. In that case, the survival probability is

$$
S(T) \approx\left[1-(\Delta H)^{2}(T / n)^{2}\right]^{n},
$$

which approaches 1 as $n \rightarrow \infty$.

It is important to note that, at this level, there should be nothing controversial or problematic about the existence of the quantum Zeno effect. This effect should be observed as long as the physical system can be made to display the behaviour shown in eq. (4). For a given system, it may be difficult or impossible to make measurements quickly enough for the quadratic time dependence of the survival probability to be observed, so that, as a practical matter, the quantum Zeno effect cannot be observed. It should be noted that the semantic arguments over terms such as 'measurement' or 'observation' can be avoided if we accept that a 'measurement' is an operation that interrupts the unitary time evolution governed by $H$ in such a way as to yield eq. (4) as a good approximation. That is, the 'measurement' should effectively act as a projection operator. According to this view, it is not necessary that the 'measurements' be recorded by a macroscopic apparatus or that they be instantaneous.

\section{The 1990 IHBW experiment}

The experiment of Itano, Heinzen, Bollinger and Wineland (IHBW) ${ }^{6}$ was based on a proposal of $\mathrm{Cook}^{7}$ for observing the quantum Zeno effect in a three-level atom (Figure 2). Levels 1 and 2 are stable on the timescale of the experiment. Level 3 decays to level 1 with the emission of a photon. In the IHBW experiment, levels 1 and 2 were two of the hyperfine sublevels of the ground ${ }^{2} S_{1 / 2}$ state of the $\mathrm{Be}^{+}$ion. Level 3 was a sublevel of the ${ }^{2} P_{3 / 2}$ excited state that decayed only to level 1 .

The experiment was carried out with a sample of about $5000 \mathrm{Be}+$ ions confined by electric and magnetic fields in a Penning trap. The steps in the experiment were as follows: (1) The ions were prepared in level 1 by optical pumping with the laser beam. (2) A resonant radio frequency (RF) magnetic field was applied for the interval required to drive the ions to level 2. (3) During the time that the RF pulse was applied, a variable number $n$ of equally spaced short laser pulses was applied to the ions (Figure 3). (4) The laser (resonant with the 1-to-3 transition) was turned on, and the induced fluorescence was recorded.

The intensity of the laser-induced fluorescence at the end of the experiment was proportional to the population of level 1. If there are no optical pulses during the long RF pulse, the population of level 2 as a function of the time interval $t$ that the RF pulse is applied is

$$
P_{2}(t)=\sin ^{2}(\Omega t / 2)=\frac{1}{2}[1-\cos (\Omega t)],
$$

where $\Omega$ is proportional to the amplitude of the RF field. If the duration of the RF pulse is chosen to be $T=\pi / \Omega$ (a pi-pulse), then all of the population is transferred from level 1 to level 2. If $n$ equally spaced laser pulses of negligible duration are applied during the RF pi-pulse, the population of level 2 at $t=T$ is

$$
P_{2}(T)=\frac{1}{2}\left[1-\cos ^{n}(\pi / n)\right],
$$

which approaches 0 as $n$ goes to infinity. 
Figure 4 compares the data to theory. The solid bars represent the transition probability as a function of $n$ according to the simplified calculation of eq. (6). The bars with horizontal stripes represent the data. The bars with diagonal stripes represent a calculation that takes into account the finite duration of the laser pulses and optical pumping effects. The data are in reasonably good agreement with the simplified calculation and in better agreement with the improved calculation. The decrease in $P_{2}(T)$ as $n$ increases demonstrates the quantum Zeno effect.

A variation of the experiment was carried out by initializing the ion in level 2 and then applying the RF field and laser pulses. In this case, transition from level 2 to level 1 was inhibited as $n$ increased. This is another example of the quantum Zeno effect, wherein inhibition of the transition is accompanied by the absence of laserinduced fluorescence.

In 2001, the quantum Zeno effect was observed for an unstable quantum system by Fischer et al. ${ }^{8}$. The quantum Zeno effect for induced transitions is not fundamentally different from that for unstable systems, since they both follow from the general arguments of Misra and Sudar$\operatorname{shan}^{2}$; but it has been difficult to observe in the latter case, because of the short time-interval over which the decay is nonexponential. Fischer et al. ${ }^{8}$ were able to create an artificial system (atoms tunnelling from a

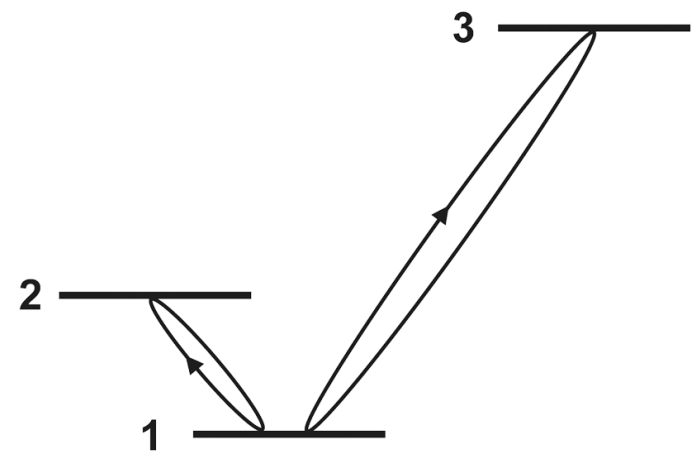

Figure 2. Energy-level diagram for the IHBW demonstration of quantum Zeno effect. A resonant field drives transitions between levels 1 and 2. Short 'measurement' pulses resonant with the transition between levels 1 and 3 interrupt the 1 -to- 2 transitions.

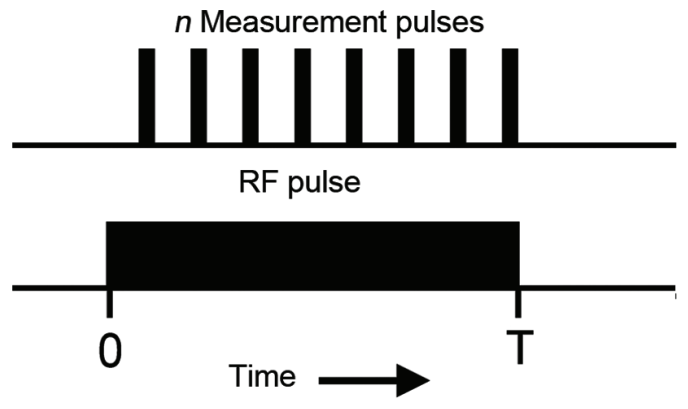

Figure 3. Timing of the radio frequency and optical fields applied to beryllium ions in the IHBW experiment. Short 'measurement' pulses resonant with the 1-to-3 transition interrupt the 1-to-2 transition driven by a single, long RF pulse. standing-wave light field) in which the interactions could be controlled so as to observe the desired effects.

\section{Responses to the IHBW experiment}

As can be seen by the history of citations (Figure 1), the publication of the IHBW experiment ${ }^{6}$ generated considerable interest. As of early November 2018, there were 757 citations $^{3}$. For a few years after publication, the number of citations per year to the IHBW paper ${ }^{6}$ was approximately equal to that number to the Misra and Sudarshan paper $^{2}$. This seems to indicate that the two papers were usually cited together, as a pair. More recently, the number of citations per year to the IHBW paper has been about half that to the Misra and Sudarshan paper. Figure 1 also shows the history of citations to the paper of Fischer et $a l^{8}{ }^{8}$. As of early November 2018, there were 263 citations $^{3}$, and the number per year is about half of the number to the IHBW paper ${ }^{6}$.

Initially, some of the responses were critical in one way or another. Ballentine ${ }^{9}$ objected to the use of the term 'wavefunction collapse' in describing the experiment. The authors responded that the concept of wavefunction collapse was not essential, and that any interpretation of quantum mechanics that yielded the same prediction of the experimental results should be regarded as valid ${ }^{10}$. Some objected to the fact that photons were not actually observed during the intermediate 'measurements', in the sense of having the scattered photons registered by a detector, so that the experiment did not actually demonstrate the quantum Zeno effect ${ }^{9,11-13}$. However, the results are predicted to be the same whether or not the intermediate measurements are made. It is enough that the measurements could have been made. As long as the laser interactions act effectively as projection operators, so that the algebra of eqs (1)-(4) is followed, the experiment should be regarded as a demonstration of the quantum Zeno effect. It should be noted that none of the criticisms was directed at the execution of the experiment itself, only at the interpretation. For the most part, citations to the

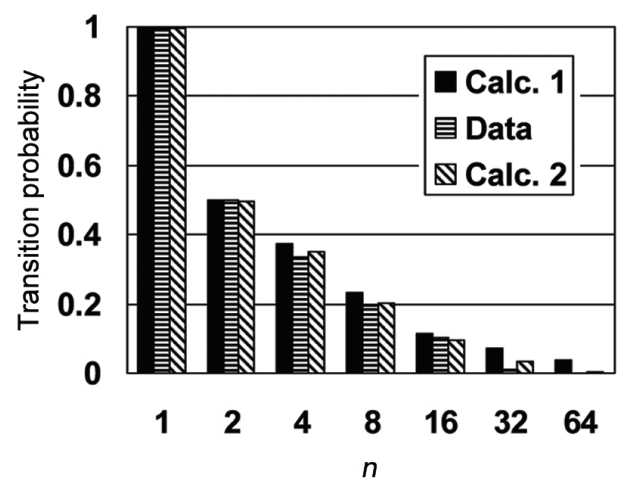

Figure 4. Probability of making the 1-to-2 transition as a function of $n$ number of optical 'measurement' pulses. Data are indicated by bars with horizontal stripes. Simple calculation (calc. 1) is indicated by solid bars and improved calculation (calc. 2) by bars with diagonal stripes. 
IHBW paper $^{6}$ simply accept it as a demonstration of the quantum Zeno effect. In fact, it is cited in quantum mechanics textbooks ${ }^{14-17}$ and popular science books ${ }^{18-22}$.

In a narrow sense, the IHBW experiment seems to have escaped criticism. That is, the execution of the experiment itself is not criticized. However, there are some who define the quantum Zeno effect in a different way than it is defined in this article and who then claim that the IHBW experiment did not verify the true quantum Zeno effect.

Here is an example, from a book by Whitaker ${ }^{18}$ :

'Having mentioned proposed experiments, it is surely time that I gave an account of a 1990 experiment which was described by its architects as demonstrating the quantum Zeno effect. I should say at the outset that this experiment which was carried out by Wayne Itano and colleagues (IHBW), and based on a proposal of Richard Cook, was brilliantly conceived and undertaken. ${ }^{18}$

Having praised the experiment, Whitaker then goes on to question whether the laser pulses constitute 'measurements'. He defines 'measurements' differently than they are defined in this article.

Heidi Fearn and Willis Lamb Jr (discoverer of the Lamb shift and 1955 Physics Nobel laureate) doubted the existence of the quantum Zeno effect ${ }^{23}$. They found fault with the derivation of Misra and Sudarshan ${ }^{2}$ and believed that the IHBW experiment could not have verified it. They do not, however, find technical fault with the experiment, only with the interpretation. Their somewhat amusing reference to the IHBW experiment does not actually address the details of the experiment:

'Ordinarily, a published paper on the quantum theory of measurement (referring to the paper of Misra and Sudarshan ${ }^{2}$ ) remains in decent obscurity. Unfortunately, some very good experiments in optical physics were performed at NIST, in Boulder, Colorado, and their authors claimed that their results confirmed the existence of the Zeno effect. The present authors strongly disagreed with this claim. ${ }^{, 23}$

This passage seems to credit (or blame) the IHBW experiment for bringing attention to the paper of Misra and Sudarshan. There might be some truth to that allegation.

On a more positive, but also amusing note, a discussion of the IHBW experiment is included in a book, by Orzel $^{22}$. The book is made up of imaginary conversations between a dog and his owner, relating to quantum physics. An entire chapter is devoted to the quantum Zeno effect. An analogy is made between probability amplitudes oscillating between the atomic states in the IHBW experiment and a hidden bunny moving inside a box.

\section{Conclusion}

The 1977 publication of Misra and Sudarshan ${ }^{2}$ stimulated a great deal of theoretical and experimental work that has enhanced our understanding of the time development of quantum systems, such as the short-time nonexponential decay of unstable quantum systems. The results of the IHBW experiment ${ }^{6}$, published in 1990, confirmed the existence of the quantum Zeno effect for the case of the inhibition of an induced transition. Interest in the quantum Zeno effect continues to be high, partially due to the possibility of practical applications in quantum information processing.

1. Wikipedia, Quantum Zeno effect. https://en.wikipedia.org/wiki/ Quantum Zeno effect (accessed on 2 November 2018).

2. Misra, B. and Sudarshan, E. C. G., The Zeno's paradox in quantum theory. J. Math. Phys., 1977, 18, 756-763.

3. Clarivate Analytics, Web of Science; https://webofknowledge.com (accessed on 2 November 2018).

4. Itano, W. M., Perspectives on the quantum Zeno paradox. J. Phys. Conf. Ser., 2009, 196, 012018.

5. Peres, A., Zeno paradox in quantum theory. Am. J. Phys., 1980, 48, 931-932.

6. Itano, W. M., Heinzen, D. J., Bollinger, J. J. and Wineland, D. J., Quantum Zeno effect. Phys. Rev. A, 1990, 41, 2295-2300.

7. Cook, R. J., What are quantum jumps? Phys. Scr., 1988, T21, 4951.

8. Fischer, M. C., Gutiérrez-Medina, B. and Raizen, M. G., Observation of the quantum Zeno and anti-Zeno effects in an unstable system. Phys. Rev. Lett., 2001, 87, 040402.

9. Ballentine, L. E., Comment on \quantum Zeno effect. Phys. Rev. A, 1991, 43, 5165-5167.

10. Itano, W. M., Heinzen, D. J., Bollinger, J. J. and Wineland, D. J., Reply to "Comment on "Quantum Zeno effect"'. Phys. Rev. A, 1991, 43, 5168-5169.

11. Petrosky, T., Tasaki, S. and Prigogine, I., Quantum Zeno effect. Phys. Lett. A, 1990, 151, 109-113.

12. Inagaki, S., Namiki, M. and Tajiri, T., Possible observation of the quantum Zeno effect by means of neutron spin-flipping. Phys. Lett. A, 1992, 166, 5-12.

13. Pascazio, S., Namiki, M., Badurek, G. and Rauch, H., Quantum Zeno effect with neutron spin. Phys. Lett. A, 1993, 179, 155-160.

14. Griffiths, D. J., Introduction to Quantum Mechanics, PrenticeHall, Englewood Cliffs, NJ, USA, 1995.

15. Sakurai, J. J. and Tuan, S. F., Modern Quantum Mechanics, Revised edition, Addison-Wesley, Reading, MA, USA, 1994.

16. Peres, A., Quantum Theory: Concepts and Methods, Kluwer Academic, Dordrecht, The Netherlands, 1993.

17. Braginsky, V. B. and Khalili, F. Y., Quantum Measurement, Cambridge University Press, Cambridge, UK, 1992.

18. Whitaker, A., Einstein, Bohr, and the Quantum Dilemma, Cambridge University Press, Cambridge, UK, 1996.

19. Coveney, P. and Highfield, R., The Arrow of Time, Fawcett Columbine, New York, USA, 1990.

20. Schulman, L. S., Time's Arrows and Quantum Measurement, Cambridge University Press, Cambridge, UK, 1997.

21. Ghose, P., Testing Quantum Mechanics on New Ground, Cambridge University Press, Cambridge, UK, 1999.

22. Orzel, C., How to Teach Physics to Your Dog. Scribner, New York, USA, 2009.

23. Fearn, H. and Lamb, Jr W. E., Comments on the quantum Zeno effect. J. Opt. B, 1995, 7, 211-214.

doi: $10.18520 / \mathrm{cs} / \mathrm{v} 116 / \mathrm{i} 2 / 201-204$ 